\title{
Pre-filter design for exact linearisation-based tracking controllers for variable-load systems
}

\author{
Na Wang ${ }^{1}$, Bálint Kiss ${ }^{1}$ \\ ${ }^{1}$ Budapest University of Technology and Economics, Müegyetem rakpart 3, 1111 Budapest, Hungary
}

\section{ABSTRACT}

Several classes of weight-handling equipment with nonlinear dynamics are exactly linearisable by feedback. This implies that a controller ensuring the exponential decay of the tracking error along a reference trajectory can be also designed. The control of both fully actuated robotic arms and underactuated crane-like systems can be addressed using this method. However, the value of model parameters such as the mass of the load or the friction coefficients must be known in order to calculate the actuated inputs in closed loop. The uncertainty of these parameters may result in unstable closed-loop dynamics or in the deterioration of the tracking performance. This paper presents a design procedure to take into consideration such uncertainties using a serial pre-filter, which is determined using $H_{\infty}$ techniques. The procedure is applied to a SCARA-type robotic arm and to a two-dimensional overhead crane system. The simulation results show the applicability of the suggested method.

\section{Section: RESEARCH PAPER}

Keywords: exact linearisation; robust design; parameter uncertainty, SCARA robot; overhead crane

Citation: Na Wang, Balint Kiss, Pre-filter design for exact linearization-based tracking controllers for variable-load systems, Acta IMEKO, vol. 8, no. 4, article 11, December 2019, identifier: IMEKO-ACTA-08 (2019)-04-11

Editor: Yvan Baudoin, International CBRNE Institute, Belgium

Received January 16, 2019; In final form September 12, 2019; Published December 2019

Copyright: This is an open-access article distributed under the terms of the Creative Commons Attribution 3.0 License, which permits unrestricted use, distribution, and reproduction in any medium, provided the original author and source are credited.

Funding: This work was supported by the BME-Artificial Intelligence FIKP grant of EMMI (BME FIKP-MI/SC).

Corresponding author: Na Wang, e-mail: wang@iit.bme.hu

\section{INTRODUCTION}

Robotic arms and cranes serve mostly as weight-handling devices, and they move a load to a desired target location along a specified path within the workspace of the equipment. A shared property of such weight-handling tasks is the uncertainty of the load mass or inertia, as load variation from one operation cycle to another may be high. Therefore, both for robots and for cranes, one of the objectives of the controller design is the limitation or full cancellation of the effects of this uncertainty on the accuracy of reference trajectory tracking.

In contrast with fully actuated robotic arms, cranes are underactuated mechanisms, such that the number of configuration variables is larger than the number of actuators. Nevertheless, both robots and cranes share the formalism (Euler-Lagrange equations) available to obtain their dynamical models.

From a control point of view, it can be observed that tracking precision and speed are generally conflicting requirements, especially if uncertainty is present for inertia values, since these uncertainties are amplified due to the high-motion speeds of the mechanism. Moreover, in addition to the load inertia, friction coefficients and torque constants of the (usually electric) actuators are also known with only a limited accuracy.

Closed-loop control is expected to ensure robust stability and tracking performance in the presence of such uncertainties.

Various methods have been already explored for robot controller design and implementation, including sliding mode control [1]-[3], neural networks [4], and fuzzy systems [5], [6], to name a few. A comprehensive overview of the robust control techniques of robot manipulators is presented in [7].

Similarly, several control techniques are proposed for cranes to ensure the tracking of a reference load trajectory. A review is presented in [8]. The flatness-based modelling and control of crane systems similar to that considered in this paper are addressed in [9] and [10]. The authors of [11] suggested robust controllers for the approximated linear crane dynamics obtained by (idle) setpoint linearisation.

Setpoint linearisation remains a valid approximation for idle positions. This limits the applicability of the feedback to slow (or quasi-static) load motions. In the case of quick load trajectories, 


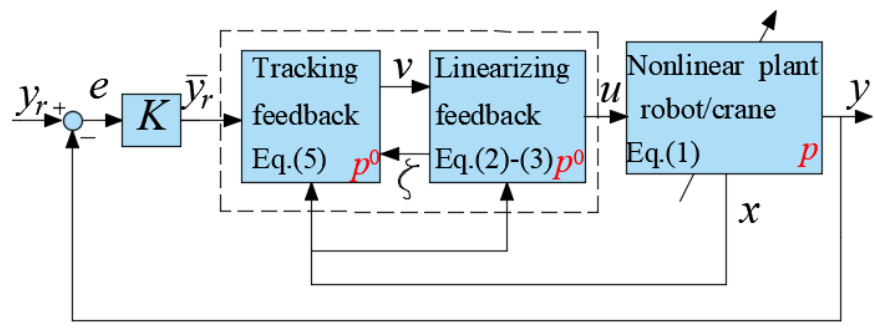

Figure 1. Closed-loop dynamics with a serial pre-filter, tracking and linearising feedback.

where the nonlinear coupling effects and the rope angles become large, the linear approximation is no longer valid.

Dynamical models of robots and cranes are differentially flat [12]; hence, they are feedback linearisable. The linearising feedback is static for robots and dynamic for cranes (i.e. the feedback has a non-empty state vector) [9], [13]. Controllers exploiting the flatness property are capable of quick and precise reference tracking if the parameters are known with accuracy. Uncertainty in parameters, such as inertia, load mass, and friction coefficients, may destabilise the closed-loop system or considerably deteriorate its performance in terms of both steadystate and transient behaviour.

This paper addresses the problem of the uncertainty of parameters by the design of a serial and linear pre-filter using standard $H_{\infty}$ synthesis techniques. The need for the robust approach is motivated by the fact that the parameters of the real system and the parameters used in the linearising feedback may be different, especially as far as the load mass is concerned. To define the underlying synthesis problem, the set of systems at which the exact linearisation is applied with a parameter misfit to the robot or crane model must be covered by a standard linear uncertainty structure. The weighting matrices of this structure are calculated by solving a set of inequalities such that the linearising feedback is applied to a large set of robot or crane models with the parameter values spanning a grid of their uncertainty range.

The idea of applying a robustifying controller together with exact feedback linearisation has already been presented by the authors in [14]. The necessary design steps to obtain the pre-filter are presented in Section 2. The suggested method is applied to a SCARA-type robot in Section 3. In Section 4, we discuss how the method can be used in crane control. The concluding section enumerates some topics subject to further investigation.

\section{THE ROBUSTIFYING PRE-FILTER AND ITS DESIGN}

Consider a nonlinear system with a finite dimensional statespace so that its description depends on the $N$ elements of a parameter vector $p=\left[p_{1}, p_{2}, \ldots, p_{N}\right]^{T}$. The uncertainty of each parameter is described by a bounded real interval $p_{i} \in Q_{i} \subset \mathbb{R}$ such that $Q=Q_{1} \times Q_{2} \times \cdots \times Q_{N}$. The vector of the nominal parameter values is denoted by $p^{0} \in \mathcal{Q}$. The nonlinear dynamics are described by

$$
\dot{x}=f(x, u, p) \quad y=h(x, p)
$$

where $x \in \mathbb{R}^{n}$ and the dimension $m$ of the input vector $u$ equal the dimension of the output vector $y$. We suppose that a linearising feedback can be designed in Equation (1) for all possible parameter values in $Q$, such that the linearising (or flat)

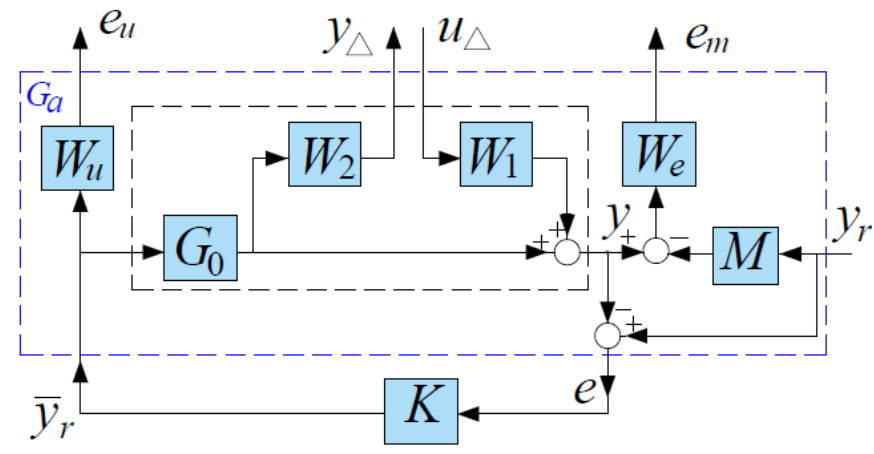

Figure 2. The controller $K(s)$ with the augmented plant.

output is $y$. Since the real parameter values are not known, the nominal values $p^{0}$ are used in the expressions of the feedback:

$\dot{\zeta}=\varphi\left(\zeta, x, v, p^{0}\right)$

$u=\psi\left(\zeta, x, v, p^{0}\right)$.

Thanks to the exact linearisability (or differential flatness property [12]) of the system, it is possible to express the derivatives of the output trajectory as the function of the full state vector of the closed-loop system, $[x, \zeta]^{T}$ :

$y_{i}^{(j)}=h_{i, j}\left(x, \zeta, p^{0}\right) \quad j=1, \ldots, \mu_{i}-1 \quad y_{i}^{(j)}=\frac{d^{j} y_{i}}{d t^{j}}$.

Let us now denote by $\bar{y}_{r}$ a sufficiently smooth reference trajectory for the output $y$. The tracking feedback law can be than set as

$v_{i}=\bar{y}_{i, r}^{\left(\mu_{i}\right)}+\sum_{j=1}^{\mu_{i}} \lambda_{i, j}\left(\bar{y}_{i, r}^{\left(\mu_{i}-1\right)}-h_{i, \mu_{i}-j}\left(x, \zeta, p^{0}\right)\right) i=1, \ldots, m$

which results in an exponential decay of the tracking error, since, by the construction of Equation (5), $e_{i}=\bar{y}_{i, r}-y_{i}$ satisfies

$0=e_{i}^{\left(\mu_{i}\right)}+\lambda_{i, 1} e_{i}^{\left(\mu_{i}-1\right)}+\lambda_{i, 2} e_{i}^{\left(\mu_{i}-2\right)}+\ldots+\lambda_{i, \mu_{i}} e_{i}$

where the coefficients $\lambda_{i, j}$ are design parameters. They are set so that the characteristic polynomial of Equation (6) is Hurwitz stable i.e. all its roots have negative real parts.

The block diagram of the closed-loop system is depicted in Figure 1. It follows from the above discussions that the closedloop transfer between $\bar{y}_{r}$ and $y$, assuming $p=p^{0}$, reads

$\frac{Y(s)}{\bar{Y}_{r}(s)}=\operatorname{diag}_{i=1, \ldots, m}\left\{\frac{\lambda_{i, \mu_{i}}}{s^{\mu_{i}}+\lambda_{i, 1} s^{\mu_{i}-1}+\ldots+\lambda_{i, \mu_{i}}}\right\}=G_{0}(s)$.

Due to the uncertainty of the parameters, the nominal values $p^{0}$ used in the linearising feedback (Equations (2)-(3)) may be different to their real values. This parameter misfit implies that the resulting closed-loop dynamics may still include nonlinearities. For the robotic arms and crane systems considered in this article, the uncertain parameters are the inertia of the moving parts of the mechanism, the mass of the load, and the friction coefficients.

The serial pre-filter (see Figure 1) is designed using $H_{\infty}$ synthesis techniques [15]. To apply such a synthesis technique, the feedback structure following the controller $K(s)$ in Figure 1 
must be replaced by an uncertainty structure as in Figure 2. A sufficiently smooth grid is chosen to cover the uncertainty range $Q$ of the parameters. For each vertex $p^{i}$ of this grid, a linear transfer function $G^{i}(s)$ is obtained between the signals $\bar{y}_{r}$ and $y$ by a setpoint linearisation. The weighting matrices $W_{1}(s)$ and $W_{2}(s)$ of the output multiplicative structure in Figure 2 are chosen so that $G^{i}(s)=\left(1+W_{1}(s) \Delta(s) W_{2}(s)\right) G_{0}(s)$ holds true for some $\|\Delta(s)\|_{\infty} \leq 1$. The command ucover in Matlab calculates the weighting matrices accordingly if the nominal model and the frequency response data for each $G^{i}(s)$ is given. The $H_{\infty}$ design problem to obtain $K(s)$ is also solved with Matlab, such that a standard augmented plant, denoted by $G_{a}(s)$, is constructed (Figure 2) with performance weighting transfer functions $M(s), W_{e}(s)$ and $W_{u}(s)$ :

$\left[\begin{array}{llll}y_{\Delta} & e_{m} & e_{u} & e\end{array}\right]^{T}=G_{a}(s)\left[\begin{array}{c}u_{\Delta} \\ y_{r} \\ \bar{y}_{r}\end{array}\right] \quad G_{a}(s)=\left[\begin{array}{ll}P_{11} & P_{12} \\ P_{21} & P_{22}\end{array}\right]$

with

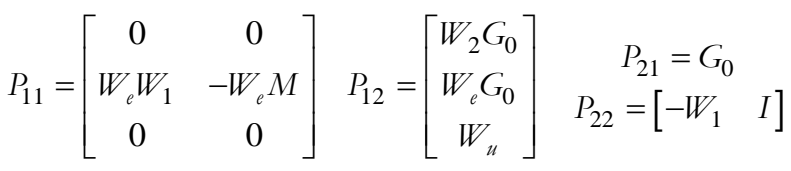

where the dependence on $s$ is omitted. Applying the controller $K(s)$, the closed-loop transfer matrix can be calculated using a lower fractional transformation

$$
F_{l}\left(G_{a}, K\right)=P_{11}+P_{12} K\left(I+P_{21} K\right)^{-1} P_{22} \text {. }
$$

By introducing $S_{o}=\left(I+G_{0} K\right)^{-1}$, we get:

$$
F_{l}\left(G_{a}, K\right)=\left[\begin{array}{cc}
-W_{2} G_{0} K S_{o} W_{1} & W_{2} G_{2} K S_{o} \\
W_{e} S_{o} W_{1} & W_{e}\left(G_{0} K S_{o}-M\right) \\
-W_{u} S_{o} W_{1} & W_{u} K S_{o}
\end{array}\right] .
$$

Finally, the $H_{\infty}$ optimisation consists of finding the controller $K(s)$, which minimises the $H_{\infty}$ norm of (11). This optimisation process is implemented in Matlab through the hinfsyn command [16]. Robust stability is also guaranteed if $\left\|W_{2} G_{0} K S_{0} W_{1}\right\|_{\infty}<1$

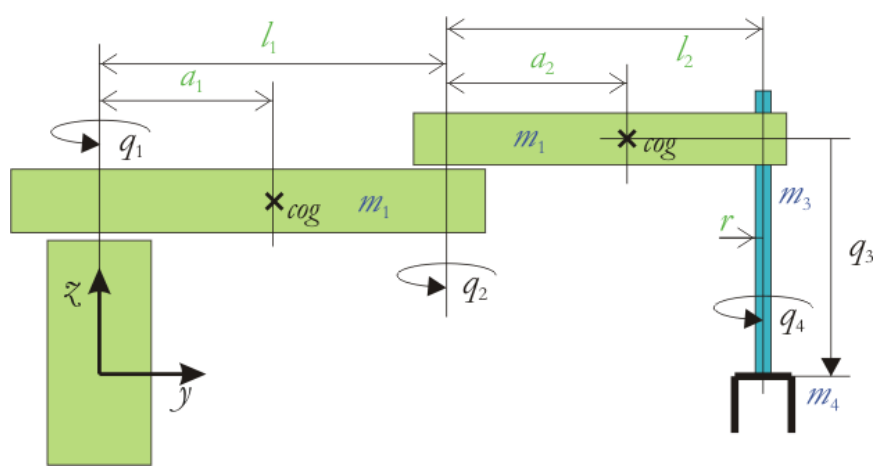

Figure 3. A SCARA robot with RRTR (R-rotational, T-translational) joint configuration.
Table 1. Uncertain parameters of the four-DOFs SCARA-type robotic arm.

\begin{tabular}{clll}
\hline Variable & Normal value & Minimum value & Maximum value \\
\hline$m_{4}$ & $3 \mathrm{~kg}$ & $2 \mathrm{~kg}$ & $4 \mathrm{~kg}$ \\
$F_{v 1}$ & $0.5 \mathrm{~kg} \mathrm{~m} \mathrm{rad}^{-1} \mathrm{~s}^{-1}$ & $0.2 \mathrm{~kg} \mathrm{~m} \mathrm{rad}^{-1} \mathrm{~s}^{-1}$ & $0.8 \mathrm{~kg} \mathrm{~m} \mathrm{rad}^{-1} \mathrm{~s}^{-1}$ \\
$F_{v 2}$ & $0.5 \mathrm{~kg} \mathrm{~m} \mathrm{rad}^{-1} \mathrm{~s}^{-1}$ & $0.2 \mathrm{~kg} \mathrm{~m} \mathrm{rad}^{-1} \mathrm{~s}^{-1}$ & $0.8 \mathrm{~kg} \mathrm{~m} \mathrm{rad}^{-1} \mathrm{~s}^{-1}$ \\
$F_{v 3}$ & $0.05 \mathrm{~kg} \mathrm{~m} / \mathrm{s}$ & $0.02 \mathrm{~kg} \mathrm{~m} / \mathrm{s}$ & $0.08 \mathrm{~kg} \mathrm{~m} / \mathrm{s}$ \\
$F_{v 4}$ & $0.05 \mathrm{~kg} \mathrm{~m} \mathrm{rad}^{-1} \mathrm{~s}^{-1}$ & $0.02 \mathrm{~kg} \mathrm{~m} \mathrm{rad}^{-1} \mathrm{~s}^{-1}$ & $0.08 \mathrm{~kg} \mathrm{~m} \mathrm{rad}^{-1} \mathrm{~s}^{-1}$ \\
\hline
\end{tabular}

The next two sections present the application of the above design procedure for a four Degrees-of-Freedom (DOF) robot arm and for a planar crane system.

\section{CONTROL OF A SCARA-TYPE ROBOTIC ARM}

\subsection{Robot modelling}

Let us consider a four-DOFs SCARA-type robotic arm with three rotary joints whose axes are parallel to each other and which have a prismatic joint. To demonstrate the feasibility of the proposed method, the design procedure described in the previous section is executed on the model of a Bosch Turbo SCARA SR 60-type robot [17]. The robot scheme is shown in Figure 3. The dynamics of a robot manipulator read

$$
H(q) \ddot{q}+C(q, \dot{q}) \dot{q}+B(\dot{q})+G(q)=\tau
$$

where $q, \dot{q}$ and $\ddot{q}$ are the vectors of joint angles, joint velocities, and joint accelerations, respectively; $H(q) \in \mathbb{R}^{4 \times 4}$ is a symmetric, positive definite inertia matrix; $C(q, \dot{q}) \dot{q} \in \mathbb{R}^{4}$ is the vector of the Coriolis and centrifugal torques; $B(\dot{q}) \in \mathbb{R}^{4}$ is the vector of the friction torques; $G(q) \in \mathbb{R}^{4}$ is the vector of gravitational torques, and the control input torque is $\tau \in \mathbb{R}^{4}$. The length, the position of the centre of gravity ( $\operatorname{cog}$ ), and the mass of the segments are denoted by $l_{i}, a_{i}$, and $m_{i}$, respectively; and $r$ is radius of the last segment. Using standard notations in robotics, $D_{i j}$ denotes the elements of $H(q)\left(D_{i j}=D_{j i}\right)$ and reminds us that

$C_{i}(q, \dot{q}) \dot{q}=\sum_{j=1}^{4} \sum_{k=1}^{4} D_{i j k} \dot{q}_{j} \dot{q}_{k}$.

Considering the SCARA robot, such that we set $a_{i}=l_{i} / 2$, the elements of the inertia matrix read

$$
\begin{aligned}
D_{11}= & \frac{m_{1} l_{1}^{2}}{3}+m_{2}\left(\frac{1}{3} l_{2}^{2}+l_{1}^{2}+l_{1} l_{2} C_{2}\right)+\frac{m_{4} r^{2}}{2}+ \\
& +\left(m_{3}+m_{4}\right)\left(l_{1}^{2}+l_{2}^{2}+2 l_{1} l_{2} C_{2}\right) \\
D_{12}= & m_{2}\left(\frac{1}{3} l_{2}^{2}+\frac{1}{2} l_{1} l_{2} C_{2}\right)+ \\
& \left(m_{3}+m_{4}\right)\left(l_{2}^{2}+l_{1} l_{2} C_{2}\right)+\frac{1}{2} m_{4} r^{2} \\
D_{14}= & -\frac{1}{2} m_{4} r^{2} \quad D_{22}=\frac{1}{3} m_{2} l_{2}^{2}+m_{3} l_{2}^{2}+m_{4}\left(l_{2}^{2}+\frac{1}{2} r^{2}\right) \\
D_{33}= & m_{3}+m_{4} \quad D_{31}=D_{32}=D_{34}=0
\end{aligned}
$$

and the non-zero $D_{i j k}$ elements are

$$
D_{112}=-\left(\frac{1}{2} m_{2}+m_{3}+m_{4}\right) l_{1} l_{2} S_{2} \quad D_{112}=2 D_{122}=-2 D_{211} .
$$


Shortcut notations $S_{2}$ and $C_{2}$ represent $\sin q_{2}$ and $\cos q_{2}$, respectively. Only viscous friction is considered as in [18]:

$B\left(\dot{q}_{i}\right)=F_{v i} \dot{q}_{i}$

and only the motion of the third joint is influenced by gravity

$$
G_{3}=-\left(m_{3}+m_{4}\right) g \quad G_{1}=G_{2}=G_{4}=0 \quad g=9.81 \mathrm{~m} / \mathrm{s}^{2} .
$$

\subsection{Pre-filter design and simulation results}

In most applications, the joint mass can only be estimated with limited accuracy. We assume that the parameters $m_{1}, m_{2}$, and $m_{3}$ are known with sufficient precision, and their values are $15 \mathrm{~kg}, 12 \mathrm{~kg}$, and $3 \mathrm{~kg}$, respectively. The parameter $m_{4}$ comprises the variable payload mass; hence, its value is uncertain. The lengths of the two links are $l_{1}=0.5 \mathrm{~m}, l_{2}=0.4 \mathrm{~m}$, and $r=0.2 \mathrm{~m}$. The friction coefficients $F_{v i}$ are also variable. The uncertain parameter ranges are given in Table 1. A grid of 50 vertices is used to get the output multiplicative uncertainty structure presented in Figure 2 with a second order weighting matrix $W_{1}$, with the following elements:

$$
\begin{array}{ll}
W_{1,11}(s)=\frac{0.27 s^{2}+0.06 s+4.61 e-4}{s^{2}+0.93 s+0.04} & W_{1,22}(s)=\frac{0.35 s^{2}+0.04 s+1.27 e-4}{s^{2}+0.56 s+0.01} \\
W_{1,33}(s)=\frac{0.45 s^{2}+0.05 s+6.09 e-4}{s^{2}+2.26 s+0.14} & W_{1,44}(s)=\frac{1.18 s^{2}+0.16 s+5.04 e-4}{s^{2}+1.45 s+0.03} \\
W_{2,11}(s)=\frac{0.71 s^{2}+0.12 s+8.13 e-4}{s^{2}+1.94 s+0.07} & W_{2,22}(s)=\frac{0.71 s^{2}+0.10 s+2.98 e-4}{s^{2}+1.06 s+0.02} \\
W_{2,33}(s)=\frac{0.45 s^{2}+0.05 s+6.09 e-4}{s^{2}+2.26 s+0.14} & W_{2,44}(s)=\frac{0.72 s^{2}+0.11 s+3.49 e-4}{s^{2}+1.07 s+0.02} .
\end{array}
$$

A reference model is used to define the desired closed-loop tracking response for each joint angle:

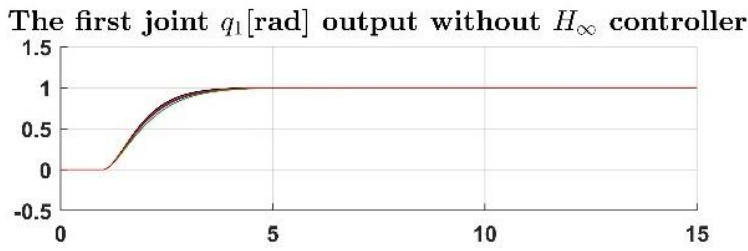

The second joint $q_{2}[\mathrm{rad}]$ output without $H_{\infty}$ controller

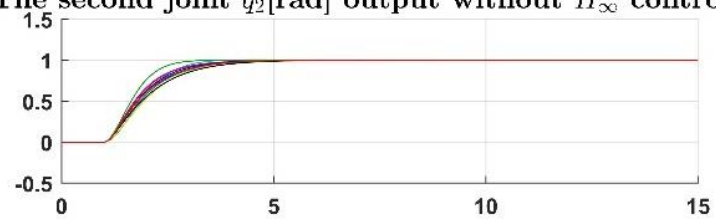

The third joint $q_{3}[\mathrm{rad}]$ output without $H_{\infty}$ controller

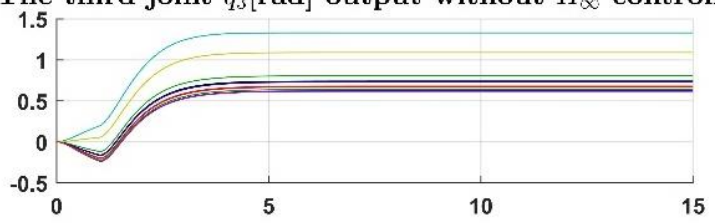

The fourth joint $q_{4}[\mathrm{rad}]$ output without $H_{\infty}$ controller

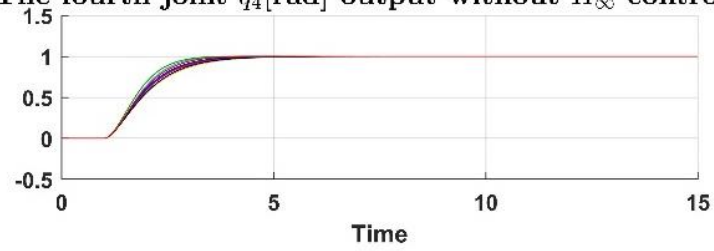

Figure 4. Joint trajectories without the $H_{\infty}$ pre-filter
$M(s)=\operatorname{diag}\left\{\frac{\omega_{m}^{2}}{s^{2}+2 \xi_{m} \omega_{m} s+\omega_{m}^{2}}\right\}$

with $\omega_{m}=2 \mathrm{rad} / \mathrm{sec}$ and $\xi_{m}=0.8$, respectively. The remaining performance weighting transfer matrices are also diagonal (fourby-four). Recall that $W_{u}$ is linked to the actuator bandwidth. The weighting functions used in our design read

$W_{u}(s)=\operatorname{diag}\left\{\frac{40\left(s+10 \omega_{m}\right)^{2}}{\left(s+200 \omega_{m}\right)^{2}}\right\}$

and

$W_{e}(s)=\operatorname{diag}\left\{W_{e 1}, 10 W_{e 1}, \frac{9}{20} W_{e 1}, 10 W_{e 1}\right\} \quad W_{e 1}=\frac{2000 \omega_{m}}{s+2 \omega_{m}}$.

The robot dynamics and the controller are simulated for different uncertain parameter values using Matlab and Simulink. The reference trajectory $y_{r}$ is a step function at $t=1 \mathrm{sec}$ for each joint. The initial conditions are set to zero. Figure 4 shows the tracking performance without the use of the pre-filter, whereas Figure 5 shows the joint trajectories with the use of the pre-filter. For each joint, a family of curves is presented, each for a different vertex of the grid spanned over the uncertain parameter space. It can be observed that the use of the pre-filter reduces the effect of uncertainty and provides better performance. Since $\left\|W_{2} G_{0} K S_{0} W_{1}\right\|_{\infty}=0.5025$, the robust stability condition (12) is satisfied.

\section{CRANE CONTROL}

\subsection{Crane modelling}

We consider a 2D overhead crane [9], depicted in Figure 6. The load is suspended by a massless rope, which is pulled by a

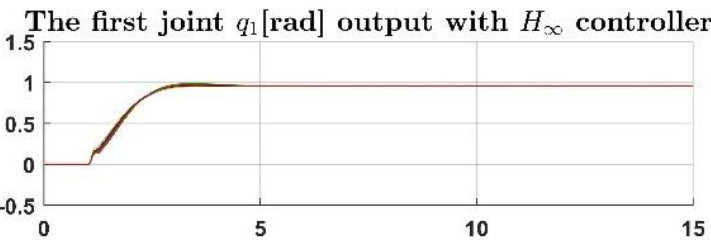

The second joint $q_{2}[\mathrm{rad}]$ output with $H_{\infty}$ controller

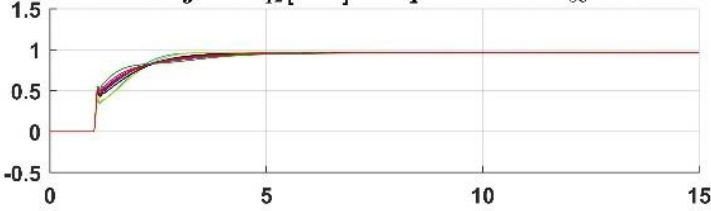

The third joint $q_{3}[\mathrm{rad}]$ output with $H_{\infty}$ controller

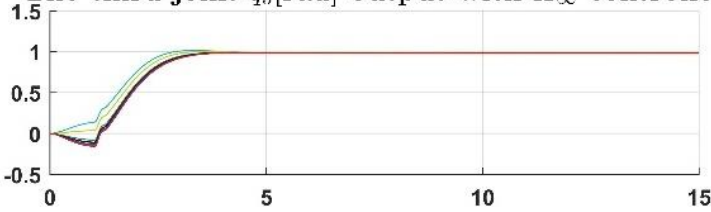

The fourth joint $q_{4}[\mathrm{rad}]$ output with $H_{\infty}$ controller

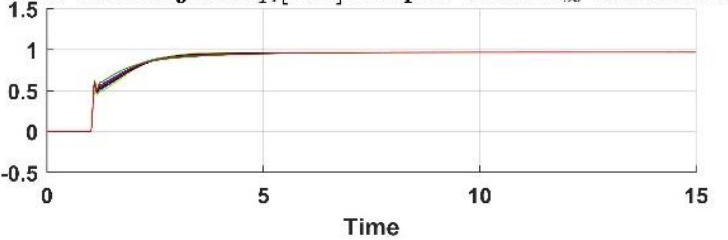

Figure 5. Joint trajectories with the application of the $H_{\infty}$ pre-filter. 


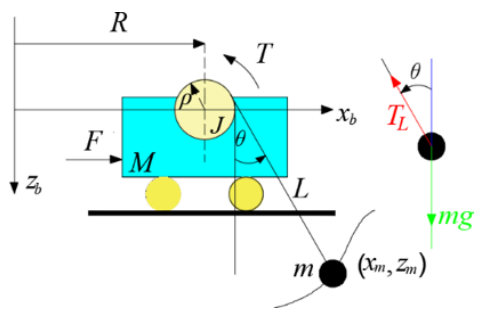

Figure 6. Overhead crane in 2D and forces acting on the load.

winch. The suspension point is moved horizontally by a cart. The configuration variables are the horizontal position of the cart $R$, the length of the rope $L$, and its angle with respect to (w.r.t.) the vertical position: $\theta$. The position of the load in the vertical $x-z$ plane is denoted by $\left[x_{m}, z_{m}\right]^{T}$ and $x=[R, L, \theta, \dot{R}, \dot{L}, \dot{\theta}]^{T}$. The parameters of the system are $p=[m, M, J, \rho]^{T}$, including the mass of the load, the mass of the cart, the inertia and the radius of the winch. Let $T=\tau / \rho$ denote the equivalent pulling force on the rope such that the system inputs are $u=[F, T]^{T}$. Let us denote the rope tension by $T_{L}$. Introducing $C_{\theta}=\cos \theta$ and $S_{\theta}=\sin \theta$, the dynamics read

$\left[\begin{array}{ccc}M+m & m S_{\theta} & m L C_{\theta} \\ m S_{\theta} & m+\frac{J}{\rho^{2}} & 0 \\ m L C_{\theta} & 0 & m L^{2}\end{array}\right]\left[\begin{array}{c}\ddot{R} \\ \ddot{L} \\ \ddot{\theta}\end{array}\right]+$

$$
+\left[\begin{array}{c}
2 m \dot{L} \dot{\theta} C_{\theta}-m L \dot{\theta}^{2} S_{\theta} \\
-m L \dot{\theta}^{2}-g m C_{\theta} \\
m L\left(2 \dot{L} \dot{\theta}+g S_{\theta}-\dot{R} \dot{\theta} S_{\theta}\right)
\end{array}\right]=\left[\begin{array}{c}
F \\
-T \\
0
\end{array}\right] .
$$

\subsection{Pre-filter design and simulation results}

It is known [9], [13] that Equation (23) is differentially flat and hence feedback linearisable. The coordinates of the load form the flat output:

$$
y_{1}=x_{m}=R+\rho+L S_{\theta} \quad y_{2}=z_{m}=L C_{\theta} \text {. }
$$

The control objective is to follow a reference trajectory specified for the flat output. The crane system can be transformed by feedback into two separated chains of integrators

$$
y_{1}^{(4)}=v_{1} \quad y_{2}{ }^{(4)}=v_{2} \text {. }
$$

The linearising feedback has two states, $\zeta_{1}$ and $\zeta_{2}$. These are the rope tension $\zeta_{1}=T_{L}$ and its first time derivative $\zeta_{2}=\dot{T}_{L}$. Let us now calculate the linearising feedback resulting (25). Referring to [19], the motion equations of the load read

$\ddot{y}_{1}=\ddot{x}_{m}=-\frac{T_{L}}{m} \sin \theta \quad \ddot{y}_{2}=\ddot{z}_{m}=-\frac{T_{L}}{m} \cos \theta+g$.

Let us differentiate both sides of (26) twice w.r.t. the time so that we use the two states of the feedback $\left(\zeta_{1}\right.$ and $\left.\zeta_{2}\right)$ as well

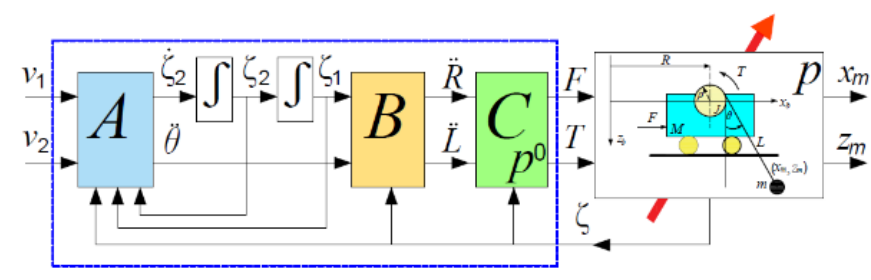

Figure 7. Calculation blocks of the flatness-based exact linearisation of the crane system.

$$
\begin{aligned}
& \frac{1}{m}\left(-\dot{\zeta}_{2} \sin \theta-2 \zeta_{2} \dot{\theta} \cos \theta-\zeta_{1} \ddot{\theta} \cos \theta+\zeta_{1} \dot{\theta}^{2} \sin \theta\right)=v_{1} \\
& \frac{1}{m}\left(-\dot{\zeta}_{2} \cos \theta+2 \zeta_{2} \dot{\theta} \sin \theta+\zeta_{1} \ddot{\theta} \sin \theta+\zeta_{1} \dot{\theta}^{2} \cos \theta\right)=v_{2}
\end{aligned}
$$

and isolate $\dot{\zeta}_{2}$ and $\ddot{\theta}$ using the nominal $m^{0}$. We get

$$
\left[\begin{array}{l}
\dot{\zeta}_{2} \\
\ddot{\theta}
\end{array}\right]=\left[\begin{array}{cc}
-S_{\theta} & -\zeta_{1} C_{\theta} \\
-C_{\theta} & \zeta_{1} S_{\theta}
\end{array}\right]^{-1}\left(\left[\begin{array}{l}
m^{0} v_{1} \\
m^{0} v_{2}
\end{array}\right]-\left[\begin{array}{c}
\zeta_{1} \dot{\theta}^{2} S_{\theta}-2 \zeta_{2} \dot{\theta} C_{\theta} \\
\zeta_{1} \dot{\theta}^{2} C_{\theta}+2 \zeta_{2} \dot{\theta} S_{\theta}
\end{array}\right]\right)
$$

Equation (24) can be differentiated twice more to get

$$
\begin{aligned}
\ddot{R}+\ddot{L} S_{\theta}+2 \dot{L} \dot{\theta} C_{\theta}+L \ddot{\theta} C_{\theta}+L \dot{\theta}^{2} S_{\theta} & =\frac{\zeta_{1}}{m} S_{\theta} \\
\ddot{L} C_{\theta}-2 \dot{L} \dot{\theta} S_{\theta}-L \ddot{\theta} S_{\theta}-L \dot{\theta}^{2} C_{\theta} & =\frac{-\zeta_{1}}{m} C_{\theta}+g
\end{aligned}
$$

which allows us to isolate the vector of accelerations as

$$
\begin{aligned}
{\left[\begin{array}{c}
\ddot{R} \\
\ddot{L}
\end{array}\right]=} & {\left[\begin{array}{cc}
1 & S_{\theta} \\
0 & C_{\theta}
\end{array}\right]^{-1}\left(\frac{1}{m^{0}}\left[\begin{array}{cc}
-S_{\theta} & -L C_{\theta} \\
C_{\theta} & L S_{\theta}
\end{array}\right]\left[\begin{array}{c}
\zeta_{1} \\
\ddot{\theta}
\end{array}\right]+\right.} \\
& \left.+\left[\begin{array}{c}
L \dot{\theta}^{2} S_{\theta}-2 \dot{L} \dot{\theta} C_{\theta} \\
g+L \dot{\theta}^{2} C_{\theta}+2 \dot{L} \dot{\theta} S_{\theta}
\end{array}\right]\right)
\end{aligned}
$$

The calculations are also illustrated in Figure 7. Blocks A, B, and $C$ represent calculations Equation (28), Equation (30), and the first two lines of Equation (23), respectively. Recall that the states of the linearising feedback, $\zeta_{1}$ and $\zeta_{2}$, must also be initialised. For the idle initial load position $\zeta_{1}(0)=m^{0} g$ and $\zeta_{2}(0)=0$. In addition to the linearising feedback, a tracking controller is also designed. Suppose that a sufficiently smooth trajectory is given for the motion of the load as $\bar{x}_{m, r}(t), \bar{z}_{m, r}(t)$ and their derivatives, such that $\bar{y}=\left[\bar{x}_{m, r}, \bar{z}_{m, r}\right]^{T}$. The tracking errors are defined as $e_{x}=\bar{x}_{m, r}-x_{m}$ and $e_{z}=\bar{z}_{m, r}-z_{m}$. The expression of the tracking controller can be obtained by specifying the desired exponential decay of the tracking errors as

$e_{x}^{(4)}+\sum_{i=0}^{3} \lambda_{x, i} e_{x}^{(i)}=0 \quad e_{z}^{(4)}+\sum_{i=0}^{3} \lambda_{z, i} e_{z}^{(i)}=0$

Since $e_{x}^{(4)}=\bar{x}_{m, r}^{(4)}-x_{m}^{(4)}=\bar{x}_{m, r}^{(4)}-v_{1} \quad$ and $\quad e_{z}^{(4)}=\bar{z}_{m, r}^{(4)}-v_{2}$, Equation (31) can be rewritten as

$v_{1}=x_{m, r}^{(4)}+\sum_{i=0}^{3} \lambda_{x, i} e_{x}^{(i)} \quad v_{2}=z_{m, r}^{(4)}+\sum_{i=0}^{3} \lambda_{z, i} e_{z}^{(i)}$

The design parameters $\lambda_{x, i}$ and $\lambda_{z, i}(i=0, \ldots, 3)$ define the roots of the characteristic equations of Equation (31), hence 


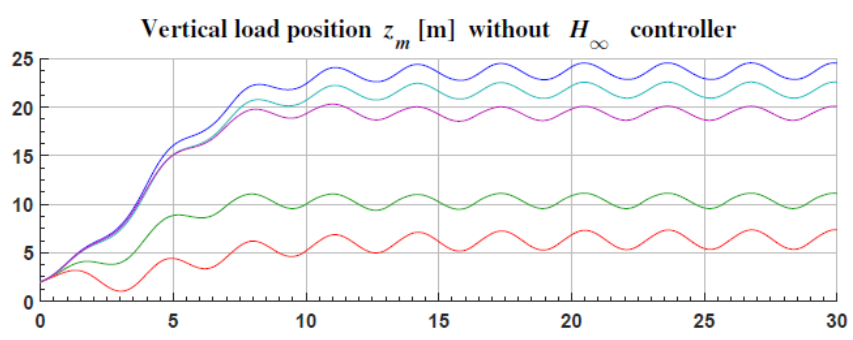

Vertical load position $z_{m}[\mathrm{~m}]$ with $H_{\infty}$ controller

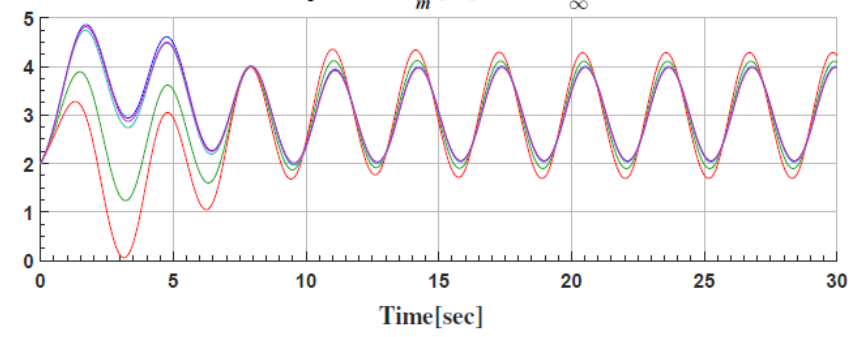

Figure 8. Vertical displacement of the load.
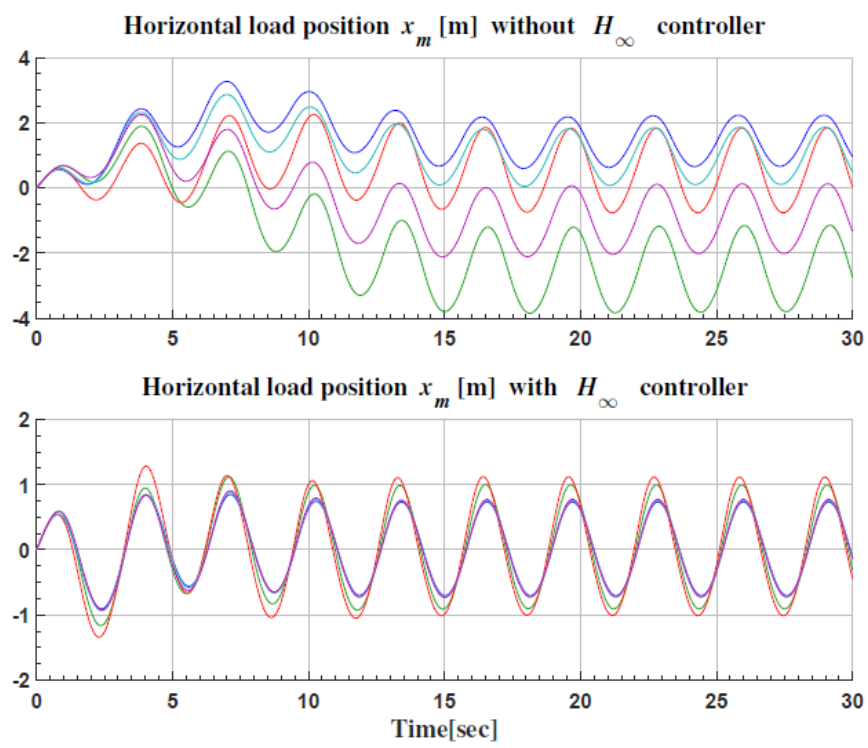

Figure 9. Horizontal displacement of the load.
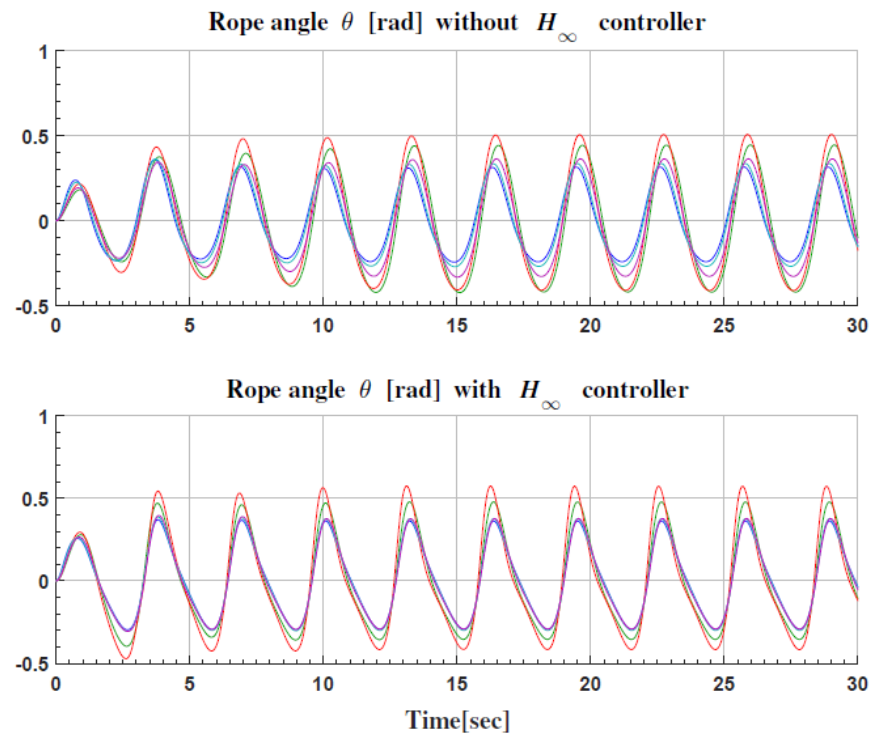

Figure 10. Rope angle.
Table 2. Uncertain and fixed parameters of the 2D crane system.

\begin{tabular}{cllc}
\hline Variable & Normal value & Minimum value & Maximum value \\
\hline$m$ & $1 \mathrm{~kg}$ & $1 \mathrm{~kg}$ & $1.6 \mathrm{~kg}$ \\
$M$ & $0.3 \mathrm{~kg}$ & $0.2 \mathrm{~kg}$ & $0.6 \mathrm{~kg}$ \\
$J$ & $0.001 \mathrm{~kg} \mathrm{~m}^{2}$ & $0.001 \mathrm{~kg} \mathrm{~m}^{2}$ & $0.001 \mathrm{~kg} \mathrm{~m}^{2}$ \\
$\rho$ & $0.03 \mathrm{~m}$ & $0.03 \mathrm{~m}$ & $0.03 \mathrm{~m}$ \\
\hline
\end{tabular}

the decay rate of the tracking errors. To realise Equation (32), one must specify how to calculate $\dot{x}_{m}, \ddot{x}_{m}, x_{m}^{(3)}, \dot{x}_{m}, \ddot{x}_{m}, z_{m}^{(3)}$ from $x$ and $\zeta$ :

$$
\begin{aligned}
\dot{x}_{m} & =\dot{R}+\dot{L} S_{\theta}+L \dot{\theta} C_{\theta} & \dot{z}_{m} & =\dot{L} C_{\theta}-L \dot{\theta} S_{\theta} \\
\ddot{x}_{m} & =-\frac{\zeta_{1}}{m^{0}} S_{\theta} & \ddot{z}_{m} & =\frac{-\zeta_{1}}{m^{0}} C_{\theta}+g \\
x_{m}^{(3)} & =-\frac{\zeta_{2} S_{\theta}+\zeta_{1} \dot{\theta} C_{\theta}}{m^{0}} & z_{m}^{(3)} & =\frac{\zeta_{1} \dot{\theta} S_{\theta}-\zeta_{2} C_{\theta}}{m^{0}} .
\end{aligned}
$$

Let us emphasise that the exponential decay of the tracking errors satisfies Equation (30) only if the parameter values used in the expressions of the feedback fit the parameters of the controlled system. The method suggested in Section 2 is applied to the crane model. The parameters and their uncertainty range are given in Table 2. A grid of 50 vertices has been spanned over the uncertain parameter space $Q$. It is possible to choose $W_{2}(s)=I$, and $W_{1}(s)$ reads

$W_{1}(s)=\operatorname{diag}\left\{\frac{0.5 s^{2}+0.005 s+1.9 e-5}{s^{2}+14.57 s+0.21}, \frac{0.32 s^{2}+0.01 s+2 e-6}{s^{2}+0.7913 s+0.72}\right\}$.

The closed-loop reference model $M(s)$ is set similarly to Equation (20), with $\omega_{m}=0.5 \mathrm{rad} / \mathrm{sec}$ and $\xi_{m}=0.8$. The $2 \times 2$ performance weighting transfer matrices $W_{e}(s)$ and $W_{u}(s)$ are set as

$W_{e}(s)=\operatorname{diag}\left\{\frac{8000 \omega_{m}}{s+2 \omega_{m}}\right\} W_{u}(s)=\operatorname{diag}\left\{\frac{500\left(s+10 \omega_{m}\right)^{2}}{\left(s+200 \omega_{m}\right)^{2}}\right\}$.

Let $\left[x_{m 0}, z_{m 0}\right]^{T}=[0,2]^{T}$ be the initial position of the reference load trajectory, which is defined as a periodic sinusoidal motion in both the $x$ and the $z$ directions

$y_{r 1}(t)=x_{m, r}(t)=\sin \left(\omega_{x} t\right) \quad y_{r 1}(t)=z_{m, r}(t)=3-\cos \left(\omega_{z} t\right)$

with $\omega_{x}=\omega_{z}=2 \mathrm{rad} / \mathrm{sec}$. This reference trajectory results in sufficiently high rope angles staying away from the region of quasi-static motions. The derivatives of the reference trajectory that are required in Equation (31) are easily calculated. The closed-loop responses are shown in Figure 8 - Figure 12 for the five representative parameter values in $Q$. All figures show the transients without (top) and with (bottom) the robustifying controller $K(s)$. We get $\left\|W_{2} G_{0} K S_{o} W_{1}\right\|_{\infty}=0.2021$, hence, the robust stability condition Equation (12) is satisfied.

Figure 8 and Figure 9 show the trajectory of the load coordinates $z_{m}$ and $x_{m}$. Figure 10 depicts the rope angles. The input signals, namely the force applied to the cart $F$ and to the winch $T$, are presented in Figure 11 and Figure 12, respectively. The robustifying controller $K(s)$ provides better performance and robustness. 


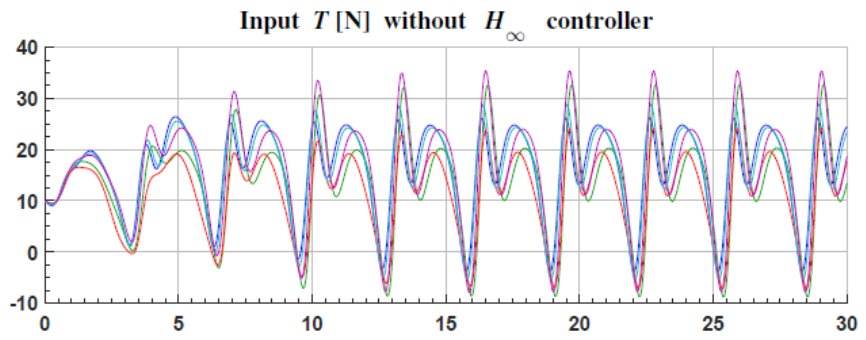

Input $T[\mathrm{~N}]$ with $H_{\infty}$ controller

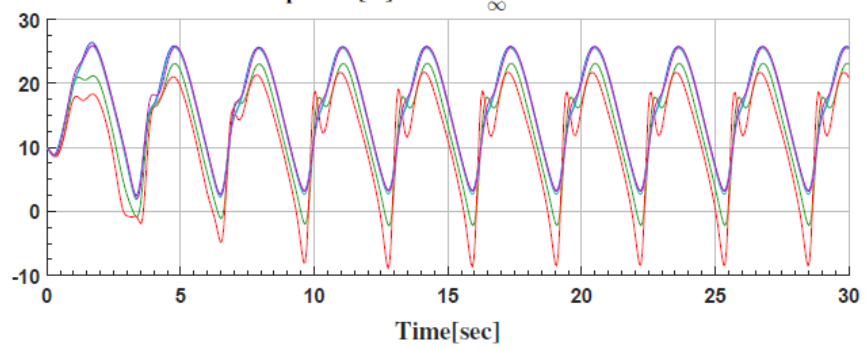

Figure 11. Input $T$ (force acting on the which) during the motion.

\section{CONCLUSIONS}

A novel method of increasing the robustness of exact linearisation against parameter uncertainty is presented for two variable-load mechanisms, namely for a fully actuated SCARAtype robot manipulator and for an underactuated 2D overhead crane. The method intends to bridge the gap between nonlinear control methods capable of stabilising fast load motions and robust synthesis techniques, ensuring robust stability and performance despite model uncertainties. As suggested by the simulation results, the proposed serial pre-filter may improve stability and performance robustness for quick reference trajectories.

Some issues may be further investigated. A restriction of the presented methods is the necessity to know all elements of the state vector $x$. If these measurements are only partly available, some observers need to be applied [20], [21].

Our studies show that the choice of the nominal values of the parameters $p^{0}$ influences the uncertainty weighting functions $W_{1}(s)$ and $W_{2}(s)$; hence, it affects the overall performance of the closed-loop system as well. The selection of the best $p^{0}$ can be investigated as an optimisation problem.

A single $K(s)$ controller may not be suitable if the uncertainty range $Q$ is too large. A possible solution for the large parameter uncertainty is the application of a gain scheduling or some adaptation techniques following the ideas presented in [22]-[24].

\section{REFERENCES}

[1] M. W. Spong, S. Hutchinson, M. Vidyasagar, Robot Modeling and Control, Wiley, 2005, ISBN: 978-0-471-64990-8.

[2] N. Adhikary, C. Mahanta, Sliding mode control of position commanded robot manipulators, Control Eng. Pract. 81 (2018), pp. 183-219.

[3] C. J. Fallaha, M. Saad, H. Y. Kanaan, K. Al-Haddad, Sliding-mode robot control with exponential reaching law, IEEE Trans. Ind. Electron. 58 (2011) pp. 600-610.

[4] L. Jin, S. Li, J. Yu, J. He, Robot manipulator control using neural networks: a survey, Neurocomputing 285 (2018) pp. 23-34.
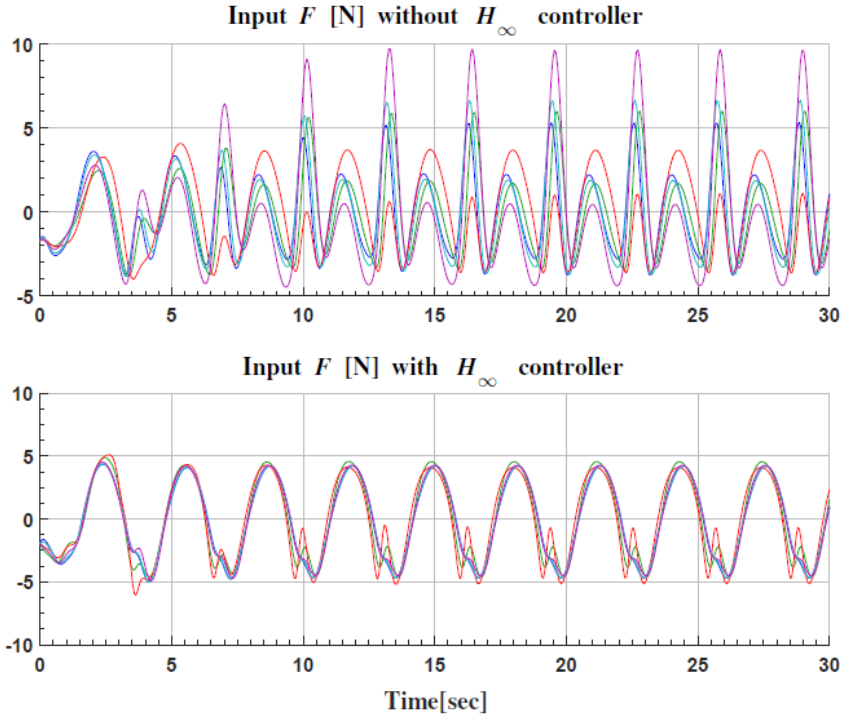

Figure 12. Input $F$ (Force acting on the cart) during the motion.

[5] K. Lochan, B. K. Roy, Control of two-link 2-DOF robot manipulator using fuzzy logic techniques: a review, Advances in Intelligent Systems and Computing 335 (2015) pp. 499-511.

[6] J. L. Meza, V. Santibáñez, R. Soto, M. A. Llama, Fuzzy self-tuning PID semiglobal regulator for robot manipulators, IEEE Trans. Ind. Electron. 59 (2012) pp. 2709-2717.

[7] H. G. Sage, M. de Mathelin, E. Ostertag, Robust control of robot manipulators: a survey, Int. J. Control 72 (2010) pp. 1498-1522.

[8] L. Ramli, Z. Mohamed, A. M. Abdullahi, H. I. Jaafar, I. M. Lazim, Control strategies for crane systems: a comprehensive review, Mechanical Systems and Signal Processing 95 (2017) pp. 1-23.

[9] B. Kiss, J. Lévine, P. Müllhaupt, Modelling, flatness and simulation of a class of cranes, Period. Polytech. Electr. Eng. 43 (1999) pp. 215-225.

[10] B. Kolar, H. Rams, K. Schlacher, Time-optimal flatness based control of a gantry crane, Control Engineering Practice 60 (2017) pp. 18-27.

[11] M. Hičár, J. Ritók, Robust crane control, Acta Polytech. Hungarica 3 (2006) pp. 91-101.

[12] J. Lévine, Analysis and Control of Nonlinear Systems. Springer, 2009. ISBN 978-3-642-00839-9.

[13] J. Lévine, P. Rouchon, G. Yuan, C. Grebogi, B. R. Hunt, E. Kostelich, E. Ott, J. A. Yorke, On the control of US Navy cranes, Proc. of the 1997 European Control Conference, 1-7 July 1997, Brussels, Belgium, pp. 2829-2833.

[14] N. Wang, B. Kiss, A robustness study of process control loops designed using exact linearization, Proc. of the $21^{\text {st }}$ International Conference on Process Control, 6-9 June 2017, Strbske Pleso, Slovakia, pp. 7-11.

[15] K.-Z. Liu, Y. Yao, Robust control: theory and applications. Wiley, 2016, ISBN: 978-1-118-75437-5.

[16] D. W. Gu, P. Petkov, M. M. Konstantinov, Robust Control Design with MATLAB. Springer, 2013, ISBN 978-1-4471-4681-0.

[17] G. Incerti, Trajectory tracking for SCARA robots with compliant transmissions: a technique to improve the positioning precision, Proc of the $12^{\text {th }}$ World Congress in Mechanism and Machine Science, 17-21 June 2007, Besancon, France, pp. 1-9.

[18] A. Mohammadi, M. Tavakoli, H. J. Marquez, F. Hashemzadeh, Nonlinear disturbance observer design for robotic manipulators, Control Eng. Pract. 21 (2013) pp. 253-267.

[19] M. Fliess, J. Lévine, P. Martin, P. Rouchon, Flatness and defect of nonlinear systems: introductory theory and examples, International Journal of Control 61 (1995) pp. 1327-1361.

[20] T. Rózsa, B. Kiss, Tracking control for two-dimensional overhead crane: feedback linearization with linear observer, Proc. of the $8^{\text {th }}$ 
International Conference on Informatics in Control, Automation, and Robotics, 28-31 June 2011, Noordwijkerhout, The Netherlands, pp. 427-432.

[21] B. Patartics, B. Kiss, Application of laser slot sensors for the state estimation of overhead cranes, Proc. of the $20^{\text {th }}$ International Conference on Process Control, 9-12 June 2015, Strbske Pleso, Slovakia, pp. 387-392.

[22] F. Gao, D. Dang, S. E. Li, M. Zhou, Control of large model mismatch systems using multiple models, Int. J. Control. Autom. Syst. 15 (2017) pp. 1494-1506.
[23] P. Zhao, R. Nagamune, Switching LPV controller design under uncertain scheduling parameters, Automatica 76 (2019) pp. 243250.

[24] L. Chen, Y. Jia, Output feedback tracking control of flat systems via exact feedforward linearization and LPV techniques, Int. J. of Control, Automation, and Systems 17 (2019) pp. 606-616. 